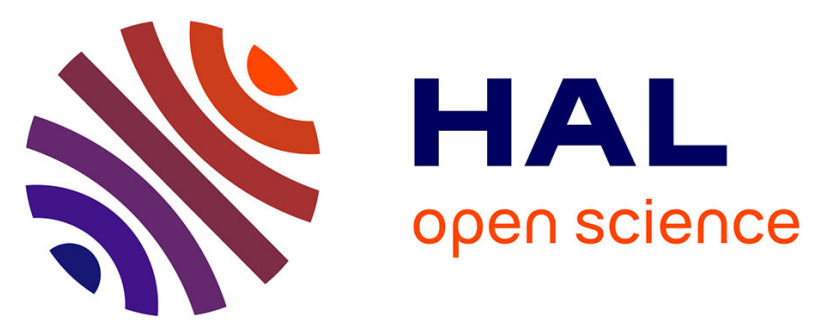

\title{
Percolation and permeability of fracture networks in excavated damaged zones
}

\author{
Valeri V. Mourzenko, Jean-François Thovert, P. M. Adler
}

\section{To cite this version:}

Valeri V. Mourzenko, Jean-François Thovert, P. M. Adler. Percolation and permeability of fracture networks in excavated damaged zones. Physical Review E: Statistical, Nonlinear, and Soft Matter Physics, 2012, 86 (2), 10.1103/PhysRevE.86.026312 . hal-01196684

\section{HAL Id: hal-01196684 \\ https://hal.science/hal-01196684}

Submitted on $10 \mathrm{Jul} 2020$

HAL is a multi-disciplinary open access archive for the deposit and dissemination of scientific research documents, whether they are published or not. The documents may come from teaching and research institutions in France or abroad, or from public or private research centers.
L'archive ouverte pluridisciplinaire HAL, est destinée au dépôt et à la diffusion de documents scientifiques de niveau recherche, publiés ou non, émanant des établissements d'enseignement et de recherche français ou étrangers, des laboratoires publics ou privés. 


\title{
Percolation and permeability of fracture networks in excavated damaged zones
}

\author{
V. V. Mourzenko* and J.-F. Thovert ${ }^{\dagger}$ \\ Institut PPRIME, CNRS-UP-ENSMA, SP2MI, BP 30179, F-86962 Futuroscope Chasseneuil Cedex, France \\ P. M. Adler \\ UPMC Sisyphe. Boîte 105, 4 Place Jussieu, F-75252 Paris Cedex 05, France
}

(Received 3 June 2012; published 22 August 2012)

\begin{abstract}
Generally, the excavation process of a gallery generates fractures in its immediate vicinity. The corresponding zone which is called the excavated damaged zone (EDZ), has a larger permeability than the intact surrounding medium. Therefore, some of its properties are of crucial importance for applications such as the storage of nuclear wastes. Field observations suggest that the fracture density is an exponentially decreasing function of the distance to the wall and that the fracture orientation is anisotropic and well approximated by a Fisher law whose pole is orthogonal to the wall. Numerical samples are generated according to these prescriptions and their percolation status and hydraulic transmissivity are systematically determined for a wide range of decay lengths and anisotropy parameters. All the numerical data are presented and discussed. A heuristic analytical expression for the percolation threshold is proposed which unifies and accurately represents all the numerical data. A simple parallel flow model yields an explicit analytical expression for the transmissivity as a function of the density, heterogeneity, and anisotropy parameters; the model also successfully accounts for all the numerical data.
\end{abstract}

DOI: $10.1103 /$ PhysRevE.86.026312

PACS number(s): 47.56.+r, 47.11.-j, 91.60.Ba

\section{INTRODUCTION}

The properties of the excavated damaged zone (EDZ) are attracting more and more attention because of their potential importance in repositories of nuclear wastes. The EDZ which is induced by the excavation process may create along the galleries of the repositories a high permeability zone which could directly connect the storage area with the ground surface. Therefore, the studies of its properties are of crucial importance.

The major properties of homogeneous, isotropic, possibly polydisperse fracture networks embedded in an impermeable [1-4] or permeable [5,6] solid matrix are now relatively well understood from a theoretical point of view. Homogeneous anisotropic networks have also been considered [7,8]. A recent work [9] summarizes and extends the results of these earlier contributions. The stereological properties of uniform or heterogeneous fracture networks have been thoroughly investigated in [10], for spatially uniform or variable fracture density. However, percolation and permeability of networks with spatially varying density have only been briefly addressed in [8].

Systematic investigations and measurements are available at Mont Terri, in Refs. [11,12], for instance. The fracture frequency was measured as a function of the distance from the tunnel walls and it could be approximated by an exponential law with a characteristic length of about $0.5 \mathrm{~m}$. Moreover, in the conceptual model of [11], the fracture orientation is strongly anisotropic and most fractures are subparallel to the tunnel walls. Similar observations were made by [12] at the

\footnotetext{
*mourzenko@ensma.fr

${ }^{\dagger}$ thovert@ensma.fr

${ }^{\ddagger}$ pierre.adler@upmc.fr
}

intersection between the Gallery 04 and the EZ-G niche at Mont Terri. These observations can be used to reconstruct realistic fracture networks representative of the EDZ and to determine their properties by direct numerical simulations.

Therefore, the major objective of this paper is to study the percolation properties and the permeability of anisotropic and/or heterogeneous fracture networks. Their density is supposed to be a decreasing exponential function of the distance to the wall while their orientations are supposed to be distributed according to a Fisher law whose pole is also perpendicular to the wall. The percolation threshold and the transmissivity of the EDZ are calculated in the directions parallel to the wall.

This paper is organized as follows. Section II provides the major ingredients for the generation of anisotropic fractures of nonuniform densities, for the analysis of their percolation properties, and for the calculation of the equivalent permeability and transmissivity. It provides in addition some definitions and relations which were for most of them derived in [10], and it recalls the main results from $[9,10]$ which are used for the analysis and modelization.

The results of the numerical computations with heterogeneous fracture networks are presented in Sec. III. The percolation properties are analyzed first. A heuristic power-law model is proposed which accurately describes the results for the percolation threshold over the whole investigated range of heterogenity and anisotropy. Then, the data for the EDZ transmissivity are presented.

A simple parallel flow model is introduced in Sec. IV. It yields an explicit analytical expression for the transmissivity as a function of the heterogeneity and anisotropy parameters, and it successfully accounts for all the numerical data. Graphical tools are provided from which first estimates can be quickly and easily obtained.

Some concluding remarks are given in Sec. V. 


\section{GENERAL}

\section{A. Heterogeneous and anisotropic fracture networks}

Consider a fractured semi-infinite medium $z \geqslant 0$, representative of the EDZ near the wall at $z=0$ [see Fig. 1(a)]. According to the observations of $[11,12]$, the fracture density $\rho(x, y, z)$ (i.e., the number of fracture centers per unit volume) is assumed to decrease exponentially as a function of the distance to the wall,

$$
\rho(x, y, z)=\rho_{0} e^{-z / \ell},
$$

where $\rho_{0}$ is the density at $z=0$ and $\ell$ is a length which characterizes the exponential decay. Furthermore, the fractures are assumed to be identical plane convex polygons with area $A$, perimeter $P$, and circumscribed by a circle of radius $R$.

When positioning a fracture in space, in addition to the center position, the direction of the normal vector $\boldsymbol{n}$ has to be set, as well as the fracture orientation angle $\omega$ in its own plane [cf Fig. 1(b)]. The latter is always distributed uniformly in the interval $[0,2 \pi]$ and for isotropic networks, the normal vector $\boldsymbol{n}$ is distributed uniformly over the unit sphere.

However, observations show that many natural fracture systems have preferential fracture orientations; for instance, Ref. [11] observed that most fractures in the EDZ are subparallel to the tunnel walls. In order to describe statistically their orientations, the vectors $\mathbf{n}$ normal to the fractures are supposed here to follow the Fisher distribution [13]. It is natural to set the polar direction parallel to the $z$ axis [i.e., normal to the gallery wall (Fig. 1(b)]. Then, the azimuth $\phi$ of $\mathbf{n}$ around the pole is uniformally distributed between 0 and $2 \pi$ and the distribution of the angle $\theta$ of $\mathbf{n}$ with the $z$ axis is given by

$$
F(\theta)=\frac{\kappa \sin \theta}{2 \sinh \kappa} e^{\kappa \cos \theta}, \quad(0 \leqslant \theta \leqslant \pi),
$$

where $\kappa$ is the concentration parameter and $F(\theta) d \theta$ is the number of fractures with a polar angle in the interval $[\theta, \theta+d \theta]$.

When $\kappa=0$, the angular distribution becomes uniform on the unit sphere whereas for large $\kappa$ the normal vectors are clustered around the pole.

\section{B. Generation of fracture networks with position-dependent densities}

Fractures are generated in a cubic unit cell $L^{3}$ [see Fig. 1(a)]. Since the network is assumed to be translationally invariant along the $x$ and $y$ directions, periodicity conditions are applied along these two directions (see [2], for instance). Hence, the

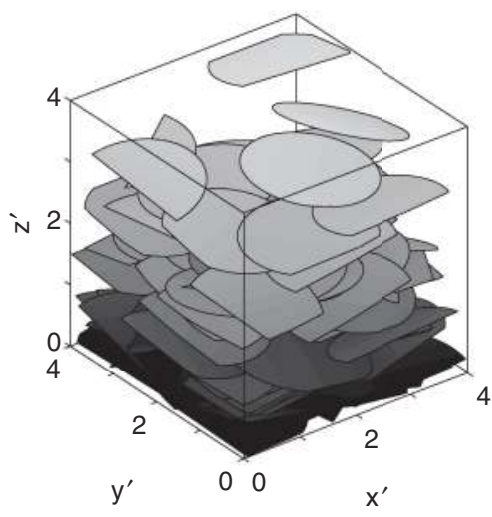

(a)

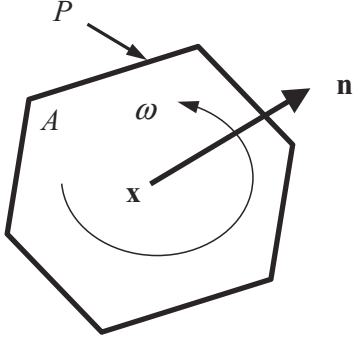

(b)

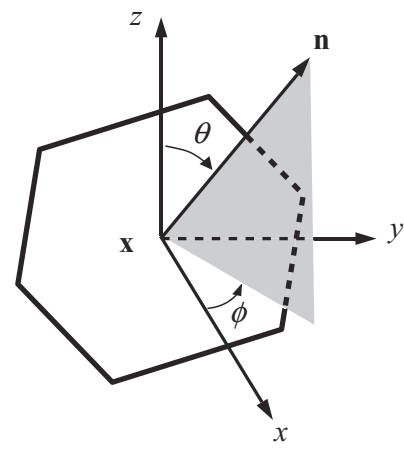

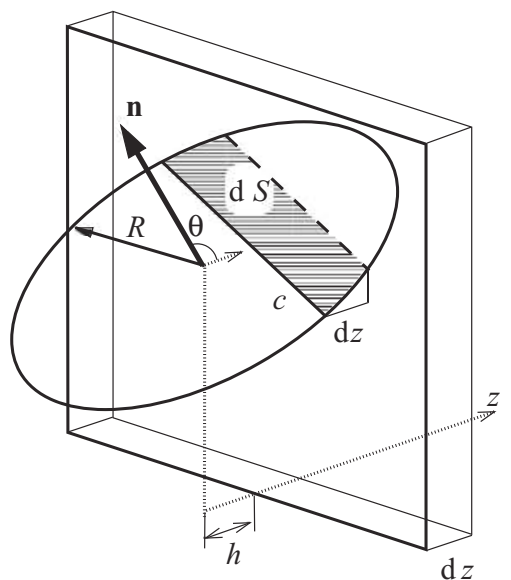

(c)

FIG. 1. Schematization of the EDZ and notations. (a) Example of an anisotropic heterogeneous network of circular fractures. The pole of the Fisher distribution (2) and the exponential variations of density (1) are aligned with the $z$ axis. (b) Notations for the fracture parameters and the system of coordinates. (c) Schematical view of a circular fracture with normal $\mathbf{n}$ intersecting the layer $\mathrm{d} z$ and producing a trace of length $c$; the contribution $d S$ to $\mathcal{S}(z)$ is the shadowed area. 
fracture centers are positioned with random values of $x$ and $y$ uniformly distributed between 0 and $L$; the fracture parts which straddle one of the cell boundaries perpendicular to the $x$ or $y$ axis, if any, are included by periodicity on the opposite side of the cell.

The $z$ direction is handled in a different way for two reasons. First, the fracture centers must be distributed along the $z$ axis in accordance with the prescribed law (1); second, the density difference at $z=0$ and $z=L$ is in contradiction with periodicity.

The parts of fractures centered at $z<0$ which intersect the plane $z=0$ are included in the cell (and similarly at the opposite side $z=L$ ). If these fractures were ignored, this would yield a lower fracture density for $0 \leqslant z \leqslant R$. This is in contradiction with the observations of [12] who found that the exponential law for the volumetric fracture area applies up to the wall.

Consequently, fractures are generated with their centers in the expanded domain $-R \leqslant z \leqslant L+R$; all the fracture parts located within the cell (i.e., between $z=0$ and $z=L$ ) are taken into account. Hence, the domain $0 \leqslant z \leqslant L$ can be regarded as a slab extracted from a larger medium where $\rho$ is given by (1). Figure 2 provides examples of generated isotropic and anisotropic fracture networks.

Throughout the rest of this paper, the lengths $L$ and $\ell$ are made dimensionless by the circumscribed radius $R$,

$$
L^{\prime}=\frac{L}{R}, \quad \ell^{\prime}=\frac{\ell}{R} .
$$

The dimensional fracture density $\rho$ is usually replaced by the dimensionless density $\rho^{\prime}$,

$$
\rho^{\prime}=\rho V_{\mathrm{ex}} \text {. }
$$

In homogeneous networks, $V_{\mathrm{ex}}$ is the excluded volume [14] which is defined as the surrounding volume into which the center of another object may not enter if overlap is to



(a)

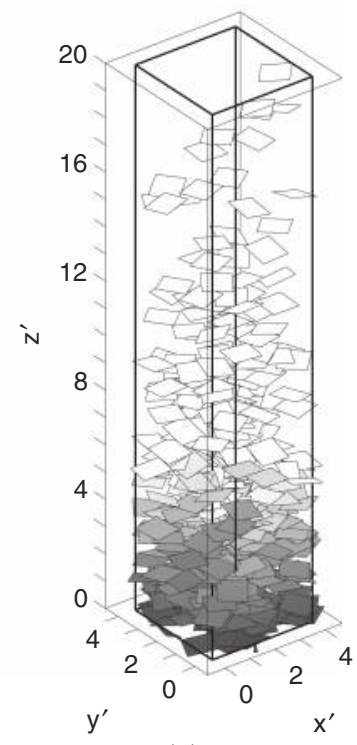

(b)
FIG. 2. Fracture networks generated with $L^{\prime}=20, l^{\prime}=5, \rho_{0}^{\prime}=$ 20 , and $\kappa=0$ (a) and 50 (b). The fractures centered in a $4 \times 4 \times 20$ volume are shown. The gray level is a decreasing function of $z^{\prime}$. be avoided. It depends on the fracture shape and on the orientation distribution. By definition, $\rho^{\prime}$ is the mean number of intersection per fractures. A correction has to be introduced to preserve this property if the density depends on position, as detailed in Sec. II C.

\section{Geometrical properties}

Since the fracture density varies with the depth $z$ measured from the wall at $z=0$, it is useful to introduce some characteristic geometrical quantities for the network in or around a plane $\Pi_{z}$ at depth $z$. These are the total trace length $\mathcal{C}(z)$ per unit surface of $\Pi_{z}$, and the area $\mathcal{S}(z)$ of fracture per unit volume in a vanishingly thin slab around $\Pi_{z}$. These two quantities are illustrated in Fig. 1(c). In particular, $\mathcal{C}(0)$ is the density of traces visible on the gallery wall, and it is the most easily accessible characteristic of the network. In addition, a generalization of the usual expression for $V_{\mathrm{ex}}$ when $\rho$ depends on position is required.

For isotropic and homogeneous networks, $\mathcal{C}, \mathcal{S}$, and $V_{\mathrm{ex}}$ were evaluated in $[15,16]$

$$
\mathcal{C}_{i}=\rho A \frac{\pi}{4}, \quad \mathcal{S}_{i}=\rho A, \quad V_{\mathrm{ex}, i}=\frac{1}{2} A P,
$$

where $A$ and $P$ are the area and the perimeter of the fractures, respectively. Correction factors can be introduced in the general case which are defined as the ratios between a given average quantity and its value for isotropic and homogeneous networks,

$$
\psi_{c}=\frac{\mathcal{C}(z)}{\mathcal{C}_{i}(z)}, \quad \psi_{s}=\frac{\mathcal{S}(z)}{\mathcal{S}_{i}(z)}, \quad \Phi=\frac{V_{\mathrm{ex}}}{V_{\mathrm{ex}, i}},
$$

where $\mathcal{C}_{i}(z)$ and $\mathcal{S}_{i}(z)$ are given by (5) with the value of $\rho$ at position $z$.

These correction factors were determined in [10] for heterogeneous and/or anisotropic networks of circular fractures,

$$
\begin{aligned}
\psi_{c}= & \frac{2 \kappa}{E \sinh \kappa}\left[I_{1}\left(\frac{E+\kappa}{2}\right) I_{o}\left(\frac{E-\kappa}{2}\right)\right. \\
& \left.+I_{o}\left(\frac{E+\kappa}{2}\right) I_{1}\left(\frac{E-\kappa}{2}\right)\right], \\
\psi_{S}= & 2 l^{\prime 2} \frac{\kappa}{\sinh \kappa}[\cosh E-\cosh \kappa],
\end{aligned}
$$

where $I_{0}$ and $I_{1}$ are the modified Bessel functions and

$$
E=\left(\kappa^{2}+\frac{1}{\ell^{\prime 2}}\right)^{1 / 2} .
$$

In the limit where the medium becomes homogeneous, these expressions tend toward

$$
\psi_{S}=1, \quad \psi_{c}=\frac{2}{\sinh \kappa} I_{1}(\kappa), \quad\left(\ell^{\prime} \rightarrow \infty\right) .
$$

Finally, $\Phi$ for anisotropic homogeneous networks is given by

$$
\Phi(\kappa, \infty)=\frac{2}{\sinh ^{2} \kappa}\left[I_{o}(2 \kappa)-\frac{1}{\kappa} I_{1}(2 \kappa)\right] .
$$


$\Phi$ could not be analytically evaluated for heterogeneous networks, but an acceptable fit is provided by

$$
\frac{\Phi\left(\kappa, \ell^{\prime}\right)}{\Phi(\kappa, \infty)}=\left[\frac{\psi_{c}\left(\kappa, \ell^{\prime}\right)}{\psi_{c}(\kappa, \infty)}\right]^{2} \quad(\kappa \gg 1) .
$$

Note that the analytical results (7) and (9) apply for any fracture shape in homogeneous networks. However, $\psi_{S}, \psi_{c}$, and $\Phi$ depend on the fracture shape in heterogeneous networks. For the square fractures considered in the present work, their values have been determined by direct measurement on the numerical samples. These data together with others for a variety of fracture shapes lead to the very pleasant conclusion that the formulas for disks are applicable to other shapes with a good accuracy if $l^{\prime}$ is replaced by $l_{A}^{\prime}$,

$$
l_{A}^{\prime}=\frac{l}{R_{A}},
$$

where $R_{A}=\sqrt{A / \pi}$ is the equivalent disk radius [10].

Since the definition (4) of the dimensionless density is kept, $\rho^{\prime}$ still represents the mean number of intersections per fracture. Moreover, a dimensionless volumetric area $\mathcal{S}^{\prime}$ and a dimensionless trace length per unit area $\mathcal{C}^{\prime}$ are defined by

$$
\mathcal{S}^{\prime}=\mathcal{S} P \Phi / 2=\rho^{\prime} \psi_{S}, \quad \mathcal{C}^{\prime}=\mathcal{C} P \Phi / 2=\frac{\pi}{4} \rho^{\prime} \psi_{C} .
$$

\section{Percolation}

Percolation in the direction orthogonal to the wall is impossible since the fracturation density decreases with $z$ and the network always becomes disconnected beyond a certain depth. Therefore, only the percolation properties of the EDZ in the directions parallel to the wall are addressed in this paper.

As usual, percolation is influenced by size effects, and samples of increasing size $L^{3}$ have to be considered in order to extrapolate the results for infinite systems, according to the procedure described in the following. However, a finite size $L$ has an additional effect, namely it truncates the EDZ in the $z$ direction. Ideally, the investigation should be conducted with a cell size $L_{z}$ in the $z$ direction large enough so that the truncated part has a negligible chance to contribute to percolation while $L_{x}$ and $L_{y}$ are varied in order to study the percolation size effects; in other words,

$$
l^{\prime} \ll L^{\prime}
$$

In practice, $L^{\prime}=5,10$, and 20 are used. Hence, Eq. (12) is a priori satisfied for small and moderate $l^{\prime}$, up to about 2 . However, for intermediate values $l^{\prime}=5 \sim 10$, it must be a posteriori checked that truncation does not influence the results. This truncation effect will be more precisely discussed when the results are presented in Sec. III C, but it is not considered anymore in this section.

Note that very large values $10^{2}$ and $10^{3}$ of $l^{\prime}$ are also considered. Therefore, the cells with $L^{\prime} \leqslant 20$ cover only the superficial part of the EDZ, and the density is nearly uniform throughout the cell. Under such circumstances, the results obtained by $[1,4]$ for three-dimensional (3D) homogeneous fracture networks are expected to be recovered.
Except for this specific point, the methodological approach of $[1,4]$ is used. For given values of $\rho_{0}^{\prime}, L^{\prime}$, and $\ell^{\prime}$, the probability $\Pi\left(L^{\prime}, \ell^{\prime}, \rho_{0}^{\prime}\right)$ of having a percolating cluster which spans the cell in the $x$ direction is calculated with $N_{r}=100$ realizations; then, the critical value $\rho_{0 c}^{\prime}\left(\ell^{\prime}, L^{\prime}\right)$ for which $\Pi=$ 0.5 is estimated. This is done by fitting the data for $\Pi\left(L^{\prime}, \ell^{\prime}, \rho_{0}^{\prime}\right)$ with a two-parameter error function of the form,

$$
\Pi\left(L^{\prime}, \ell^{\prime}, \rho_{0}^{\prime}\right)=\frac{1}{\Delta_{L} \sqrt{2 \pi}} \int_{-\infty}^{\rho_{0}^{\prime}} \exp \left\{-\frac{\left[\xi-\rho_{0 c}^{\prime}\left(\ell^{\prime}, L^{\prime}\right)\right]^{2}}{2\left(\Delta_{L}\right)^{2}}\right\} d \xi,
$$

where $\Delta_{L}$ is the width of the transition region of $\Pi\left(L^{\prime}, \ell^{\prime}, \rho_{0}^{\prime}\right)$.

In the limit of large $L^{\prime}, \rho_{0 c}^{\prime}\left(\ell^{\prime}, L^{\prime}\right)$ is expected to follow the standard scaling law [17],

$$
\rho_{0 c}^{\prime}\left(\ell^{\prime}, L^{\prime}\right)-\rho_{0 c}^{\prime}\left(\ell^{\prime}, \infty\right) \propto L^{\prime-1 / v},
$$

where $\rho_{0 c}^{\prime}\left(\ell^{\prime}, \infty\right)$ is the critical density for an infinite domain and $v$ is the critical exponent. The EDZ is a thick but essentially two-dimensional (2D) object, and it is not entirely obvious whether $v$ should be equal to the classical values $v_{2}=4 / 3$ in two dimensions, or $v_{3} \approx 0.88$ in three dimensions [17]. Since $v$ describes the divergence of the size of the connected clusters which extend in the $x$ and $y$ directions and ultimately exceed the EDZ thickness, it is reasonable to expect that the two-dimensional behavior prevails.

Percolation theory also predicts that the width $\Delta_{L}$ of the transition region scales with $L^{\prime}$ as

$$
\Delta_{L} \propto L^{\prime-1 / v} .
$$

Hence, the exponent $v$ can be determined from the data for $\Delta_{L}$ for increasing sizes $L^{\prime}$. On the other hand, by combining Eqs. (14) and (15),

$$
\rho_{0 c}^{\prime}\left(\ell^{\prime}, L^{\prime}\right)-\rho_{0 c}^{\prime}\left(\ell^{\prime}, \infty\right) \propto \Delta_{L} .
$$

Therefore, $\rho_{0 c}^{\prime}\left(\ell^{\prime}, \infty\right)$ can be determined from a linear fit of the data for $\rho_{0 c}^{\prime}\left(\ell^{\prime}, L^{\prime}\right)$ and $\Delta_{L}$ obtained in finite samples.

In practice, the percolation probability $\Pi\left(L^{\prime}, \ell^{\prime}, \rho_{0}^{\prime}\right)$ was evaluated from sets of $N_{r}=100$ realizations, in cells of sizes $L^{\prime}=5,10$, and 20 , for about 10 values of the network density evenly distributed in a range where $\Pi$ varies from 0.05 to 0.95 . The $95 \%$ confidence interval is estimated to be about \pm 0.04 in terms of $\rho_{0 c}^{\prime}(L)$.

Finally, it should be noted that it would be appropriate for such an analysis to consider parallelepipedic cells with a fixed depth $L_{z}$ equal to a few times $l$ and varying sizes $L_{x}=L_{y}$. However, the increase of $L_{z}$ by keeping it equal to $L_{x}$ and $L_{y}$ is cost free since this does not yield a significant increase of the number of fractures in the network.

The percolation threshold of homogeneous fracture networks has been studied in earlier works and the results are summarized in [4]. $\rho_{c}^{\prime}$ is nearly invariant except for very slender fracture shapes. It is in the range $2.3 \pm 0.1$ for any regular polygon. For instance, $\rho_{c}^{\prime}$ was found equal to 2.24 for triangles, 2.31 for squares and hexagons, and it seems to converge toward 2.4 in the circular limit. These results and others including very elongated shapes are well represented by the model,

$$
\rho_{c}^{\prime}=2.41\left[1-4\left(\eta-\frac{2}{\pi}\right)^{2}\right], \quad \text { with } \eta=\frac{4}{P^{\prime}} .
$$


Furthermore, it has been noted in [8] that the anisotropic character of a Fisher orientation distribution has a very weak influence on the percolation threshold if any.

\section{E. Flow properties}

Again, only the flow properties of the EDZ along the $x$ and $y$ directions are of interest when a pressure gradient $\overline{\overline{\nabla p}}$ parallel to the wall is applied. The transmissivity $\boldsymbol{T}$ which relates the total flow rate per unit width $\boldsymbol{Q}$ along the wall through the whole EDZ to the pressure gradient, is defined by

$$
\boldsymbol{Q}=-\frac{1}{\mu} \boldsymbol{T} \cdot \overline{\overline{\nabla p}},
$$

where $\mu$ is the fluid viscosity. $\boldsymbol{T}$ is homogeneous to $\left[L^{3}\right]$. Since the fracture network is statistically isotropic in the $(x, y)$ directions, $\boldsymbol{T}$ is a two-dimensional spherical tensor,

$$
\boldsymbol{T}=T \boldsymbol{I}_{\|},
$$

where $T$ is the scalar transmissivity and $\boldsymbol{I}_{\|}$the twodimensional unit tensor $\left[\boldsymbol{e}_{x} \boldsymbol{e}_{x}+\boldsymbol{e}_{y} \boldsymbol{e}_{y}\right]$.

On a local scale corresponding to the typical aperture $b$ of a fracture, the flow of a Newtonian fluid through it is governed by the Stokes equation. If $b$ is assumed to be much smaller than the typical lateral extent $2 R$ of the fracture, the flow at a scale intermediate between $b$ and $2 R$ is governed by the Darcy equation,

$$
\mathbf{q}=-\frac{1}{\mu} \sigma \overline{\nabla p},
$$

where $\mathbf{q}$ is the locally averaged flow rate per unit fracture width $\left[L^{2} T^{-1}\right], \overline{\nabla p}$ the pressure gradient, and $\sigma\left[L^{3}\right]$ the fracture conductivity coefficient. The mass conservation equation reads

$$
\nabla_{S} \cdot \mathbf{q}=0
$$

where $\nabla_{S}$ is the two-dimensional gradient operator in the fracture plane.

The medium is supposed to be spatially periodic along the $x$ and $y$ axes. In other words, identical unit cells of volume $\tau_{0}$ are juxtaposed in the plane $z=0$ and a macroscopic pressure gradient $\overline{\overline{\nabla p}}$ is applied upon the corresponding unbounded medium. Periodic boundary conditions for the local flow rate $\mathbf{q}$ and the local pressure gradient $\overline{\nabla p}$ are applied along the $x$ and $y$ axes. A no-flux condition is applied over the planes $z=0$ and $z=L$ bounding the unit cell in the third direction.

The seepage velocity $\overline{\mathbf{v}}$ can be evaluated as

$$
\overline{\mathbf{v}}=\frac{1}{\tau_{0}} \int_{S_{f}} \mathbf{q} d s,
$$

where $S_{f}$ is the surface of all the fractures in the network. $\overline{\mathbf{v}}$ is related to the pressure gradient by the Darcy law [15],

$$
\overline{\mathbf{v}}=-\frac{1}{\mu} \mathbf{K} \cdot \overline{\overline{\nabla p}} .
$$

The macroscopic permeability tensor $\mathbf{K}$ is determined from Eqs. (21) and (22) once the problem Eqs. (19) and (20) have been solved. Of course, calculations are only made for $\overline{\overline{\nabla p}}$ parallel to the $x$ and $y$ axes. $\mathbf{K}$ is a two-dimensional tensor homogeneous to the square of a length. Since the $x$ and $y$ axes are statistically equivalent, $\mathbf{K}$ is a spherical $2 \mathrm{D}$ tensor when averaged over many realizations,

$$
\mathbf{K}=K \boldsymbol{I}_{\|} \cdot
$$

The transmissivity of the $x y$-periodic domain of thickness $L$ is

$$
\boldsymbol{T}=L \mathbf{K} .
$$

If the cell size $L$ is large enough to encompass the whole EDZ, or at least its part which can significantly contribute to the flow, $\boldsymbol{T}$ is the transmissivity of the EDZ.

In this paper, $\sigma$ is taken to be constant over each fracture. Because of the classical Poiseuille law, the typical conductivity $\sigma_{0}$ of a fracture is expected to be of the order of

$$
\sigma_{0}=\frac{b^{3}}{12} .
$$

This value, together with $R$ and a reference pressure $p_{0}$, is used to recast the equations in a dimensionless form. The dimensionless parameters (with primes) are defined by

$$
\begin{aligned}
& p=p_{0} p^{\prime}, \quad \boldsymbol{x}=R \boldsymbol{x}^{\prime}, \quad \nabla=\frac{1}{R} \nabla^{\prime}, \quad \mathbf{v}=\frac{\sigma_{0} p_{0}}{\mu R^{2}} \mathbf{v}^{\prime}, \\
& \mathbf{q}=\frac{\sigma_{0} p_{0}}{\mu R} \mathbf{q}^{\prime}, \quad \sigma=\sigma_{0} \sigma^{\prime}, \quad \mathbf{K}=\frac{\sigma_{0}}{R} \mathbf{K}^{\prime}, \quad \mathbf{T}=\sigma_{0} \mathbf{T}^{\prime} .
\end{aligned}
$$

The following developments use this dimensionless formulation and the results are presented in terms of the dimensionless scalar permeability $K^{\prime}$ or transmissivity $T^{\prime}$.

The numerical method applied to solve the flow problem is described by [2]. First, the fracture network is discretized; an unstructured triangulation of the fractures is obtained by using an advancing front technique. Since the triangular mesh takes into account the fracture intersections which are randomly located, it generally contains triangles of various sizes and shapes. The maximal size of the triangles in the mesh is $\delta_{M}$ which is set equal to $R / 4$. A typical number of triangles for a square fracture is 120 . As an example, a uniform network of density $\rho^{\prime}=9.1$ in a cell of size $L^{\prime}=20$ contains $1.2710^{4}$ fractures; the corresponding mesh contains $7.2510^{5}$ nodes and $1.510^{6}$ triangles.

The pressure $p$ is determined at each node of the triangular mesh, by solving a set of linear algebraic equations resulting from a finite volume formulation. Each of these equations results from the mass balance obtained by summing up the fluxes incoming from all the triangles incident to the node, in all the fractures which contain it. These fluxes are evaluated by (19) where the pressure gradient is regarded as uniform over each triangle.

For each set of parameters, the flow simulations are performed on $N_{r}=50$ randomly and independently generated fracture networks. Unless otherwise stated, the macroscopic permeabilities given in the following are always averaged over these $N_{r}$ realizations and over the two directions $x$ and $y$. Nonpercolating networks with zero macroscopic permeability are also taken into account in the averages. Note that the correction for the discretization effects described in [9] is systematically applied.

Let us recall for later use some earlier results about the permeability of homogeneous fracture networks. Consider first 
networks of infinite fractures. Their permeability tensor $\mathbf{K}_{S}$ is given by [18]

$$
\mathbf{K}_{S}=\mathcal{S} \sigma\langle\mathbf{I}-\mathbf{n n}\rangle,
$$

where the brackets indicate an average over the fracture orientations. If the components of the vector $\mathbf{n}$ are denoted by $n_{i}(i=1,2,3), \mathbf{n n}$ is the tensor of components $n_{i} n_{j}$ $(i, j=1,2,3)$. In isotropic networks $\langle\mathbf{I}-\mathbf{n n}\rangle=2 / 3 \mathbf{I}$. This yields in dimensionless form,

$$
\mathbf{K}_{S i}^{\prime}=k_{S} \rho^{\prime} \mathbf{I}, \quad \text { with } k_{S}=\frac{4}{3 P^{\prime}} .
$$

If the fracture orientations obey the Fisher law (2), Eq. (27a) becomes

$$
\mathbf{K}_{S}^{\prime}=\frac{K_{S i}^{\prime}}{\Phi}\left(\begin{array}{ccc}
\psi_{\perp} & 0 & 0 \\
0 & \psi_{\perp} & 0 \\
0 & 0 & \psi_{\|}
\end{array}\right)
$$

where $\Phi$ is given by (9). $\psi_{\perp}$ and $\psi_{\|}$were obtained in [10] as

$$
\psi_{\perp}=\frac{3}{2}\left(1+\frac{1-\kappa \operatorname{coth} \kappa}{\kappa^{2}}\right), \quad \psi_{\|}=\frac{3}{\kappa^{2}}(\kappa \operatorname{coth} \kappa-1) .
$$

Note that $\psi_{\perp}$ corresponds to the directions normal to Fisher's pole (i.e., parallel to the gallery wall).

Snow's formula (27) is an upper asymptotic value of the permeability of very dense finite fracture systems. The permeability of homogeneous isotropic networks of finite fractures was modeled by [9] as

$$
\widehat{K}^{\prime}\left(\rho^{\prime}\right)=\frac{\alpha\left(\rho^{\prime}-\rho_{c}^{\prime}\right)^{2}}{1+\beta\left(\rho^{\prime}-\rho_{c}^{\prime}\right)} .
$$

This heuristic formula represents the very extensive set of numerical data of [9] within $\pm 6 \%$ for a variety of fracture shapes in the range $3 \leqslant \rho^{\prime} \leqslant 128$ with the parameters $\alpha=$ 0.0370 and $\beta=0.155$. However, an even better accuracy of $\pm 2 \%$ is achieved in the specific case of square fractures considered in the present work with $\alpha=0.0359$ and $\beta=$ 0.148 . Note that the ratio $\alpha / \beta$ corresponds to $k_{S}$ in Eq. (27b).

Finally, it was shown in [9] that the permeability of anisotropic networks can be expressed in a form similar to Eq. (27c) for infinite fractures, with $K_{S i}^{\prime}$ replaced by the model $\widehat{K}^{\prime}(29)$ for finite fracture networks,

$$
\mathbf{K}^{\prime}=\frac{\widehat{K}^{\prime}}{\Phi}\left(\begin{array}{ccc}
\psi_{\perp} & 0 & 0 \\
0 & \psi_{\perp} & 0 \\
0 & 0 & \psi_{\|}
\end{array}\right)
$$

Note that many of the results recalled in Secs. II D and II E have been used by [19] in his modelization of the stress-induced permeability in rock salt, where the onset of percolation was expressed in terms of a critical deviatoric stress.

\section{RESULTS FOR HETEROGENEOUS NETWORKS}

\section{A. Simulations}

Simulations are performed to study the four dimensionless parameters which govern the physical situation, namely $L^{\prime}, \ell^{\prime}$, $\rho_{o}^{\prime}$, and $\kappa$. Note that $L^{\prime}$ is an artificial parameter introduced by the finite character of any numerical cell. Three sizes $L^{\prime}=$ 5,10 , and 20 were used; $\ell^{\prime}$ ranges from 0.2 to 1000 , and the density $\rho_{0}^{\prime}$ up to 400 in some cases. Simulations for isotropic and anisotropic networks are performed with $\kappa=0,10,50$, and 200. The fractures are square, unless otherwise stated.

The maximal number of fractures generated is $2.110^{4}$. Most calculations are done on a $2.66-\mathrm{GHz}$ Xeon processor. A typical case with $1.310^{4}$ fractures lasts approximately $1 \mathrm{~h}$ of CPU time for the network generation, the intersection analysis, the percolation test, and the fracture triangulation. The flow problem with $710^{5}$ unknowns over $1.510^{6}$ mesh triangles lasts approximately $7 \mathrm{~h}$.

\section{B. Fracture surface area}

Illustrative results relative to the volumetric fracture surface area are presented here in order to validate the numerical network generation procedure and the theoretical predictions of [10] recalled in Sec II C and to illustrate the influence of the adopted conventions.

Figure 3 shows the volumetric area profiles $\mathcal{S}^{\prime}(z)$ measured in fracture networks with $L^{\prime}=20, \rho_{0}^{\prime}=20$, and $l^{\prime}=1 / 2$ or 2. $\mathcal{S}^{\prime}$ was measured by cumulating in intervals of width $R / 4$ the areas of the surface elements in the network triangulation whose barycenters lie in the corresponding interval. These data are compared with the theoretical prediction $\psi_{S} \rho^{\prime}(z)$, and with $\rho^{\prime}(z)$ which disregards the effect of heterogeneity on the volumetric area [see Eq. (11)].

For $l^{\prime}=2$, the two quantities are nearly equivalent since the correction is only about $1.4 \%$ and the numerical data agree with the prediction. However, $\psi_{S} \approx 1.25$ for $l^{\prime}=1 / 2$, which is a significant correction. The numerical data are in good agreement with this prediction.

The volumetric area profile obtained numerically for $l^{\prime}=2$ when the fractures with their centers at $z<0$ are ignored, is also displayed in Fig. 3. It shows a strong depletion near the wall at $z=0$. Note that $l^{\prime}=2$ corresponds to the EDZ studied in [12] and that no such depletion was detected.

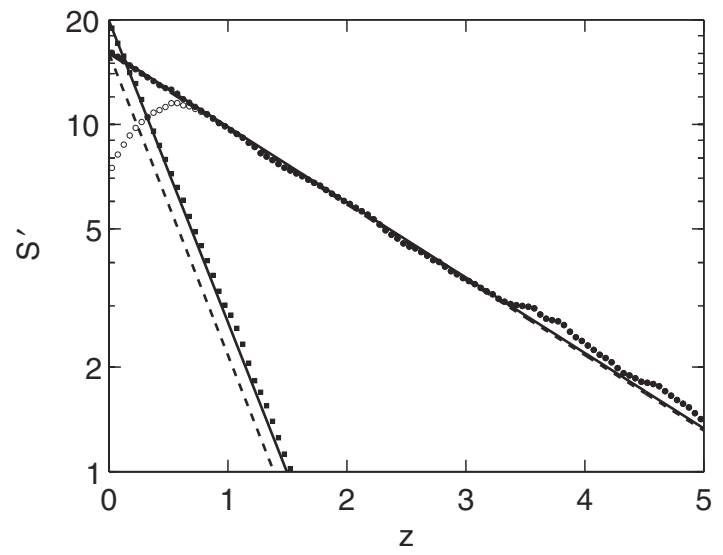

FIG. 3. Volumetric fracture surface area $\mathcal{S}^{\prime}$ as a function of depth, measured in a cell with $L^{\prime}=20$, when $\rho_{0}^{\prime}=20$ and $l^{\prime}=1 / 2$ (single realization, solid square) or $l^{\prime}=2$ (average over five realizations), with (solid circle) and without (open circle) taking into account the fractures centered at $z<0$. The solid lines are the theoretical prediction $\psi_{S} \rho^{\prime}$ [see Eq. (11)] and the broken lines are $\rho^{\prime}$. 


\section{Percolation properties}

\section{Isotropic networks}

For any prescribed decay length $l^{\prime}$, the fracture density profile is entirely defined by the degree of fracturation at $z=0$, which can be quantified by any of the parameters $\mathcal{S}_{0}^{\prime}, \mathcal{C}_{0}^{\prime}$, or $\rho_{0}^{\prime}$. In view of Eq. (11), there is a correspondence between these three measures through the known functions $\psi_{S}$ and $\psi_{c}$ of $l^{\prime}$. Hence, any of them can be used to quantify the EDZ fracturation intensity and critical values for percolation can be determined in any of these terms.

The trace density $\mathcal{C}_{0}^{\prime}$ is the most practical quantifier of the degree of fracturation since it can be directly observed on the wall. However, it will turn out that $\mathcal{S}_{0}^{\prime}$ is more appropriate when the fracture orientation distribution is anisotropic (see Sec. III C2). Therefore, the results are presented for the three parameters, namely the dimension volumetric area $\mathcal{S}^{\prime}$, trace density $\mathcal{C}^{\prime}$, and density $\rho^{\prime}$.

Examples of percolation probability curves $\Pi\left(L^{\prime}, \ell^{\prime}, \mathcal{C}_{0}^{\prime}\right)$ for some of the investigated values of $\ell^{\prime}$ and for the three cell sizes $L^{\prime}=5,10$, and 20 are given in Fig. 4. These curves have the same shapes as in standard percolation problems in spatially homogeneous fracture networks. They are shifted leftwards when $l^{\prime}$ decreases which means that a larger trace density on the wall is required for percolation when the exponential density decay in the EDZ is steeper. The width of the transition region decreases when $L^{\prime}$ increases in agreement with (15).

This transition width $\Delta_{L}$ was quantified by fitting $\Pi$ by an error function according to (13) and it is plotted in log scales against the cell size $L^{\prime}$ in Fig. 5(a). There are clearly two sets of

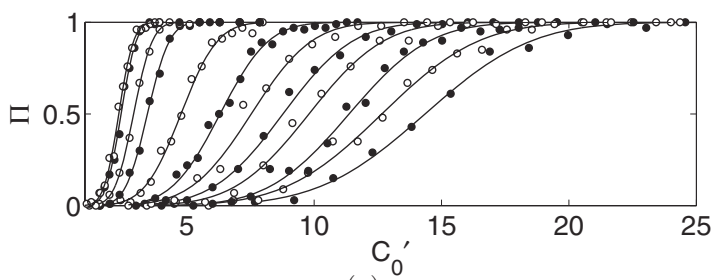

(a)



(b)

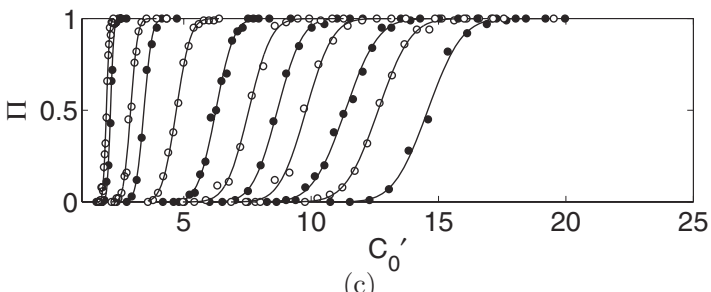

(c)

FIG. 4. Percolation probability $\Pi\left(L^{\prime}, l^{\prime}, \mathcal{C}_{0}^{\prime}\right)$ as a function of $\mathcal{C}_{0}^{\prime}$ for $L / R=5$ (a), 10 (b), and 20 (c). Data are for $\ell / R=0.2,0.25,0.3$, $0.4,0.5,0.67,1,2,5,10,100$, and 1000 , from right to left. The curves are the fits by the error function (13).
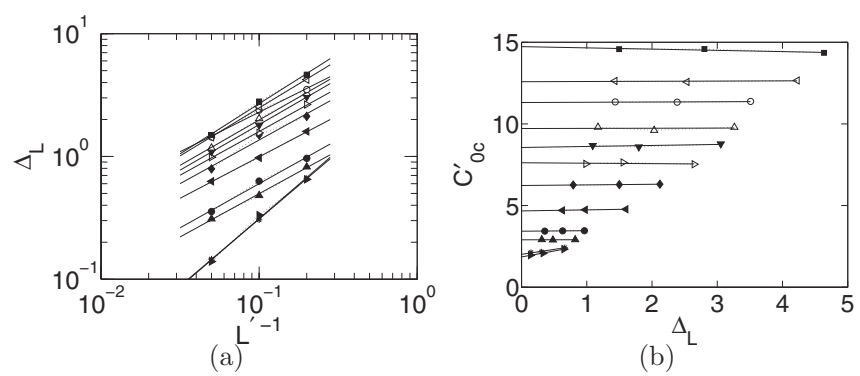

FIG. 5. Percolation properties of isotropic heterogeneous networks. (a) The transition width $\Delta_{L}$ as a function of $L^{\prime-1}$. (b) The percolation threshold $\mathcal{C}_{0 c}^{\prime}$ as a function of $\Delta_{L}$. Data are for $\ell / R=0.2$ (solid square), 0.25 (open left triangle), 0.3 (open circle), 0.4 (open up triangle), 0.5 (solid down triangle), 0.67 (open right triangle), 1 (solid diamond), 2 (solid left triangle), 5 (solid circle), 10 (solid up triangle), 100 (asterisk), and 1000 (solid right triangle). The solid lines are the linear fits.

curves which are all fairly linear, but the large values $l^{\prime}=10^{2}$ and $10^{3}$ yield a steeper slope than the small or intermediate $l^{\prime}$. A least-square fit of the form of Eq. (15) gives $v \approx 0.92$ and 0.90 for $l^{\prime}=10^{2}$ and $10^{3}$, respectively, while it ranges from 1.22 to 1.55 for $0.2 \leqslant l^{\prime} \leqslant 10$. These determinations of $v$ are not very accurate due in particular to the limited range of $L^{\prime}$, but they are sufficient to identify unambiguously two- and three-dimensional behaviors, associated with $v_{2}=4 / 3$ and $\nu_{3} \approx 0.88$ [17].

The fit of $\Pi$ by (15) also provides $\mathcal{C}_{0 c}^{\prime}\left(l^{\prime}, L^{\prime}\right)$, which is plotted versus $\Delta_{L}$ in Fig. 5(b). In view of Eq. (16), the threshold $\mathcal{C}_{0 c}^{\prime}\left(l^{\prime}, \infty\right)$ is obtained by linearly extrapolating the data for $\Delta_{L}=0$. Finally, $\mathcal{C}_{0 c}^{\prime}\left(l^{\prime}, \infty\right)$, denoted for simplicity $\mathcal{C}_{0 c}^{\prime}\left(l^{\prime}\right)$, and the corresponding $\mathcal{S}_{0 c}^{\prime}\left(l^{\prime}\right)$ and $\rho_{0 c}^{\prime}\left(l^{\prime}\right)$ are plotted in Figs. 6(a)$6(c)$ versus the decay length. They all decrease monotically as $l^{\prime}$ increases, and converge toward 1.83 for $\mathcal{C}_{0 c}^{\prime}\left(l^{\prime}\right)$ and 2.34 for $\mathcal{S}_{0 c}^{\prime}\left(l^{\prime}\right)$ and $\rho_{0 c}^{\prime}\left(l^{\prime}\right)$ when $l^{\prime} \rightarrow \infty$. This is in good agreement with the threshold values $\rho_{c}^{\prime}=2.28$ found by [1], 2.31 obtained by [4], and 2.36 predicted by (17) in homogeneous networks of square fractures.

Let us now detail some aspects of the results. Because of computational constraints, the cell depth $L^{\prime}$ could not be always kept much larger than $l^{\prime}$. Thus, with the values $L^{\prime}=5,10$, and 20 used in the analysis, three situations occur:

$$
\begin{aligned}
& l^{\prime} \ll L^{\prime}, \text { for } l^{\prime} \leqslant 2 ; \\
& l^{\prime} \sim L^{\prime}, \text { for } l^{\prime}=5 \text { or } 10 ; \\
& l^{\prime} \gg L^{\prime}, \text { for } l^{\prime}=10^{2} \text { or } 10^{3} .
\end{aligned}
$$

The first case is the simplest since no part of the EDZ with a significant chance to contribute to percolation is truncated. For instance, for the worst case $l^{\prime}=2$ and $L^{\prime}=5$, the density $\rho^{\prime}$ at $z=L^{\prime}$ is 0.48 when $\mathcal{C}_{0}^{\prime}$ is equal to $\mathcal{C}_{0 c}^{\prime}$. In other words, the truncated part of the EDZ is very sparse with less than half an intersection per fracture and it can be rightly ignored as far as percolation is concerned.

This is less obvious for Eq. (31b) which can only be checked a posteriori. Consider for instance $l^{\prime}=5$. When $L^{\prime}=5$, percolation is almost certain $(\Pi \approx 1)$ when $\mathcal{C}_{0}^{\prime}$ is equal to 4. Then, the volumetric area at $z^{\prime}=5$ is $\mathcal{C}^{\prime}(5)=1.47$. Hence, 


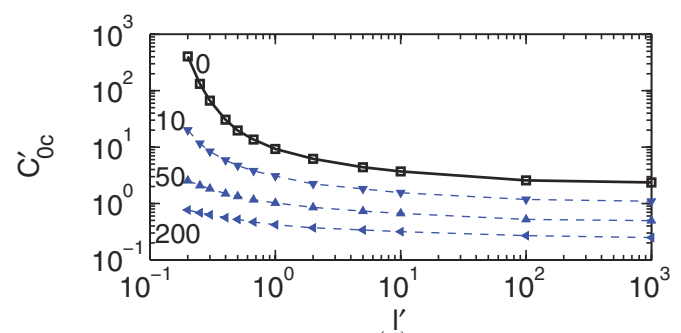

(a)

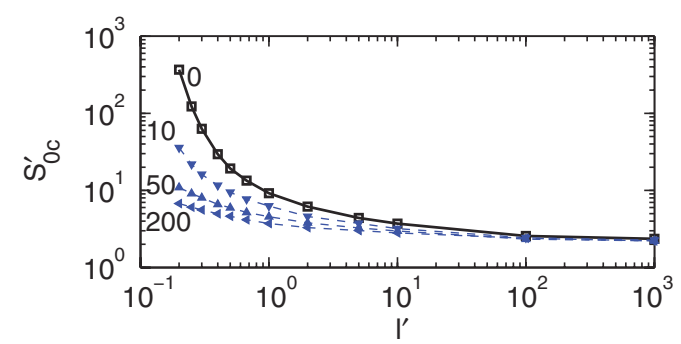

(b)

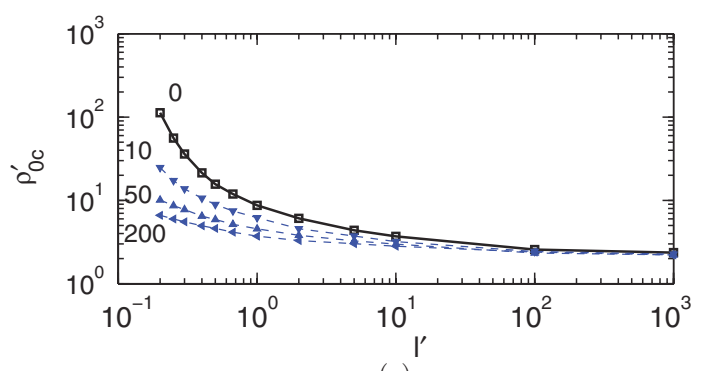

(c)

FIG. 6. (Color online) The percolation thresholds $\mathcal{C}_{0 c}^{\prime}$ (a), $\mathcal{S}_{0 c}^{\prime}$ (b), and $\rho_{0 c}^{\prime}$ (c), obtained by the extrapolation of the data to $\Delta_{L}=0$ in Fig. 5(b), as functions of $l^{\prime}$. Data are for isotropic (open square, $\kappa=0$ ), and anisotropic networks (solid circle, $\kappa=10,50$, and 200). Numbers denote the values of $\kappa$.

the probability of percolation of the truncated part of the EDZ alone is the value of $\Pi$ obtained with $\mathcal{C}_{0}^{\prime}=1.47$ which is vanishingly small (no occurrence in 100 realizations) for all the values of $L^{\prime}$. Therefore, it can be assumed that the truncation influences neither the threshold value $\mathcal{C}_{0 c}^{\prime}\left(l^{\prime}, L^{\prime}\right)$, nor the width $\Delta_{L}$ of the transition region. The same reasoning applies when $l^{\prime}=10$, for $L^{\prime} \geqslant 10$.

More generally, it can be considered that the truncation does not influence the results if the following a posteriori criterion is satisfied:

$$
e^{-L^{\prime} / l^{\prime}}\left[\mathcal{C}_{0 c}^{\prime}+\Delta_{L}\right]<\mathcal{C}_{0 c}^{\prime}-\Delta_{L} .
$$

For Eqs. (31a) and (31b), the typical size of the connected fracture clusters grows as $\mathcal{C}_{0}^{\prime}$ increases, but the growth in the $z$ direction is soon limited by the decay of the fracturation density. The cluster grows only along the $x$ and $y$ directions until it diverges and percolation occurs. Therefore, the governing exponent is $v_{2}$ as shown by Fig. 5 .

The situation is very different for Eq. (31c) with $l^{\prime} \gg L^{\prime}$ because the cluster growth remains tridimensional in the investigated range. Accordingly, the value $v_{3}$ of its scaling exponent is observed in Fig. 5. A transition to $v_{2}$ is expected if $L^{\prime}$ could be set larger than $l^{\prime}$.

The unit cell of dimensionless size $L^{\prime 3}$ is far from representative of the whole EDZ and the truncated part may significantly contribute to the percolation probability. Therefore, $\mathcal{C}_{0 c}^{\prime}$ can be overestimated. However, when $l^{\prime} \rightarrow \infty$, the EDZ tends towards a homogeneous fractured semi-infinite space and its percolation threshold in any direction should converge to the value for three-dimensional homogeneous networks (i.e., $\rho_{c}^{\prime} \approx 2.31$ [4] or $\mathcal{C}_{0 c}^{\prime} \approx 1.81$ ). This is a lower bound for $\mathcal{C}_{0 c}^{\prime}$ and the value 1.85 obtained for $l^{\prime}=10^{3}$ shows that the overestimation is very limited.

All the observations in this section are quite similar to [20] who examined percolation in thick films, regarded as lattice systems unbounded along two directions and of finite thickness $h$ in the third one. A correspondence can be made between the thickness $h$ in lattice units and the decay length $l^{\prime}$ in fracture size units. Reference [20] observed that the critical exponent $v_{3}$ prevails when considering percolation across the film (i.e., when the typical cluster size reaches $h$ ), and a transition to the two-dimensional behavior with $v_{2}$ when percolation along the film is considered. The critical site occupancy probability for percolation along the film was found to be a decreasing function of $h$; it converges toward the standard value for 3D lattices when $h \gg 1$. Although the present work addresses a continuous percolation problem, our results are qualitatively identical.

\section{Anisotropic networks}

Anisotropic networks with $\kappa=10,50$, and 200 were investigated along the same lines as isotropic networks in Sec. III C1. The results for $\mathcal{C}_{0 c}^{\prime}, \mathcal{S}_{0 c}^{\prime}$, and $\rho_{0 c}^{\prime}$ are plotted in Figs. 6(a)-6(c) as functions of the decay length. The following features are observed.

(i) The critical density remains in all cases a decreasing function of $l^{\prime}$.

(ii) It is also a decreasing function of $\kappa$.

(iii) When $l^{\prime} \rightarrow \infty, \mathcal{S}_{0 c}^{\prime}$ and $\rho_{0 c}^{\prime}$ converge for all $\kappa$ toward the value for isotropic homogeneous networks, which is an overall minimum.

(iv) Conversely, $\mathcal{C}_{0 c}^{\prime}$ remains a decreasing function of $\kappa$ even in homogeneous networks.

As recalled in Sec. II D, it has been noted in Ref. [8] that the anisotropic character of a Fisher orientation distribution does not influence the percolation threshold $\rho_{c}^{\prime}$ in homogeneous networks. The same property for $\mathcal{S}_{c}^{\prime}$ in (iii) is a consequence of $\psi_{s}=1$ in homogeneous networks regardless of $\kappa$ [see Eq. (8)]. This is not the case for $\psi_{c}$, which explains (iv).

This has the unfortunate consequence that whereas the trace density on the wall is an easily accessible quantity, it is a poor indicator for the prediction of percolation because of the sampling bias. The probability for a fracture to intersect a plane normal to the Fisher pole strongly decreases with $\kappa$. The situation would of course be different if a trace map in a plane normal to the wall were available.

The normalization of $l^{\prime}$ by $\psi_{c}$ is a heuristic but very successful way to account for the influence of $\kappa$ on $\mathcal{S}_{0 c}^{\prime}$ and $\rho_{0 c}^{\prime}$. In addition, the use of $l_{A}^{\prime}$ instead of $l^{\prime}$ also unifies the results for different fracture shapes. This is illustrated in Fig. 7 where all the data from Figs. 6(b) and 6(c) are gathered along a single curve. Data for isotropic networks of circular fractures are also shown in Fig. 7, and they are seen to fall on the same curve. 


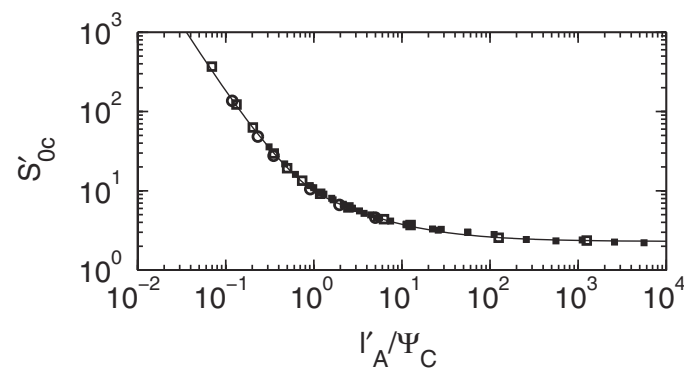

(a)

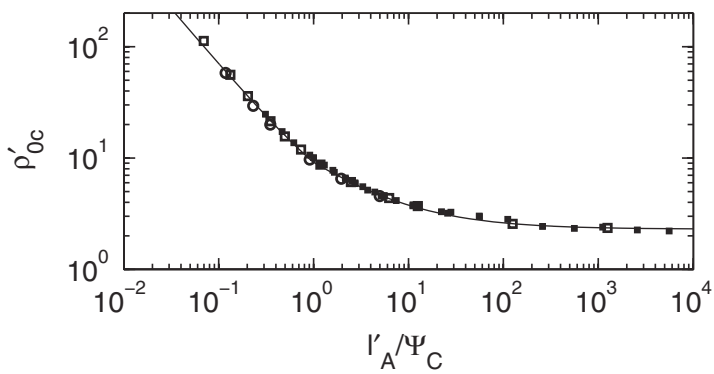

(b)

FIG. 7. The percolation thresholds $\mathcal{S}_{0 c}^{\prime}$ (a) and $\rho_{0 c}^{\prime}$ (b) as functions of $l^{\prime} / \psi_{c}$. Same data as in Figs. 6(b) and 6(c) for isotropic (open square, $\kappa=0$ ) and anisotropic networks (solid circle, $\kappa=10,50$, and 200). The lines correspond to the fit (33).

The following model can be proposed for $\rho_{0 c}^{\prime}$ :

$$
\begin{aligned}
& \rho_{0 c}^{\prime}=\rho_{c}^{\prime}+6.80\left(\frac{l_{A}^{\prime}}{\psi_{c}}\right)^{-1.002}, \quad l_{A}^{\prime} / \psi_{c} \leqslant 1, \\
& \rho_{0 c}^{\prime}=\rho_{c}^{\prime}+6.87\left(\frac{l_{A}^{\prime}}{\psi_{c}}\right)^{-0.667}, \quad l_{A}^{\prime} / \psi_{c} \geqslant 1 .
\end{aligned}
$$

These expressions result from least-square fits in log space of the data for $\rho_{0 c}^{\prime}-\rho_{c}^{\prime}$ in isotropic networks of disks and squares, with values 2.4 (disks) and 2.3 (squares) for the threshold $\rho_{c}^{\prime}$ in uniform networks and the expression (7a) in terms of $l_{A}^{\prime}$ for $\psi_{c}$. The root mean square relative deviation between the data and the model is $5.8 \%$ for Eq. (33a) and $2.8 \%$ for Eq. (33b). Furthermore, the results for anisotropic networks are also well described by Eq. (33). The overall rms relative deviation is $5.0 \%$ for the whole data set in the range $0.1 \leqslant l^{\prime} \leqslant 1000$ and $0 \leqslant \kappa \leqslant 200$.

Note that the range $l_{A}^{\prime} / \psi_{c} \geqslant 1$ for Eq. (33b) corresponds to $l^{\prime} \geqslant 1.06$ or 1.08 in isotropic networks of squares and disks, respectively. This range broadens in anisotropic networks, for instance, into $l^{\prime} \geqslant 0.52,0.24$, and 0.12 , for squares with $\kappa=$ 10,50 , and 200, respectively. Therefore, the second regime [Eq. (33b)] prevails in most practical situations.

A corresponding model for the percolation threshold in terms of $\mathcal{S}_{0 c}^{\prime}$ is obtained by multiplication of Eq. (33) by $\psi_{S}$. This is illustrated in Fig. 7(a), where the line results from the multiplication of Eqs. (33) and (7b) for $\kappa=0$. No such unifying transformation could be devised for $\mathcal{C}_{0 c}^{\prime}$.

There is an apparent contradiction in the fact that both $\mathcal{S}_{0 c}^{\prime}$ and $\rho_{0 c}^{\prime}$ are functions of $l^{\prime} / \psi_{c}$ only, since their ratio $\psi_{s}$ is a function of both $l^{\prime}$ and $\kappa$. However, it can be checked numerically that $\psi_{s}$ is also nearly a function of $l^{\prime} / \psi_{c}$ only; the values obtained for $\kappa=0$ and $\kappa=200$ with identical $l^{\prime} / \psi_{c}$
TABLE I. Calculations of the transmissivity for isotropic networks. For each decay length $l^{\prime}$, the table gives the investigated sample sizes $L^{\prime}$, the EDZ percolation threshold $\rho_{0 c}^{\prime}$, the largest investigated density $\rho_{0, \max }^{\prime}$, and the coefficients $\left(\alpha_{T}, \beta_{T}\right)$ of the fit $((34)$.)

\begin{tabular}{lccccc}
\hline \hline$l^{\prime}$ & $L^{\prime}$ & $\rho_{0 c}^{\prime}$ & $\rho_{0, \max }^{\prime}$ & $\alpha_{T}$ & $\beta_{T}$ \\
\hline 0.2 & 5 & 112.6 & 398.5 & $1.4710^{-3}$ & 1.23 \\
0.5 & 5,10 & 15.7 & 83.0 & $2.1310^{-2}$ & 1.26 \\
1.0 & $5,10,20$ & 8.72 & 45.3 & $5.2310^{-2}$ & 1.29 \\
2.0 & $5,10,20$ & 6.09 & 24.4 & $9.1910^{-2}$ & 1.34 \\
5.0 & $5,10,20$ & 4.38 & 26.2 & $1.4910^{-1}$ & 1.47 \\
10.0 & $5,10,20$ & 3.70 & 11.5 & $2.2510^{-1}$ & 1.55 \\
1000.0 & $5,10,20$ & 2.36 & 9.07 & $5.6310^{-1}$ & 1.82 \\
\hline \hline
\end{tabular}

differ by less than $5 \%$ when $l^{\prime} / \psi_{c} \geqslant 0.1$ and by less than $1 \%$ when $l^{\prime} / \psi_{c} \geqslant 0.25$. Hence, the modelization of $\rho_{0 c}^{\prime}$ as the function (33) of $l^{\prime} / \psi_{c}$ or its counterpart for $\mathcal{S}_{0 c}^{\prime}$ can be used indifferently and with an equivalent precision in the practical range of $l^{\prime}$.

\section{Transmissivities}

\section{Isotropic networks}

The transmissivity $T^{\prime}$ is investigated with the same values of the cell size $L^{\prime}$ and decay length $l^{\prime}$ as for the percolation tests, and over a wide range of densities $\rho_{0}^{\prime}$ (see Table I). For each set of the parameters $\left(l^{\prime}, L^{\prime}, \rho_{0}^{\prime}\right), T^{\prime}$ is calculated along the $x$ and $y$ directions, in 50 random realizations of the networks. The data presented hereafter are the averages over these $2 \times 50$ results.

The raw results are shown in Fig. 8 as functions of $\rho_{0}^{\prime}$ for the largest $L^{\prime}$ available for each value of $l^{\prime}$. In addition, data for smaller $L^{\prime}$ are also shown for $l^{\prime}=5$ and 1000.

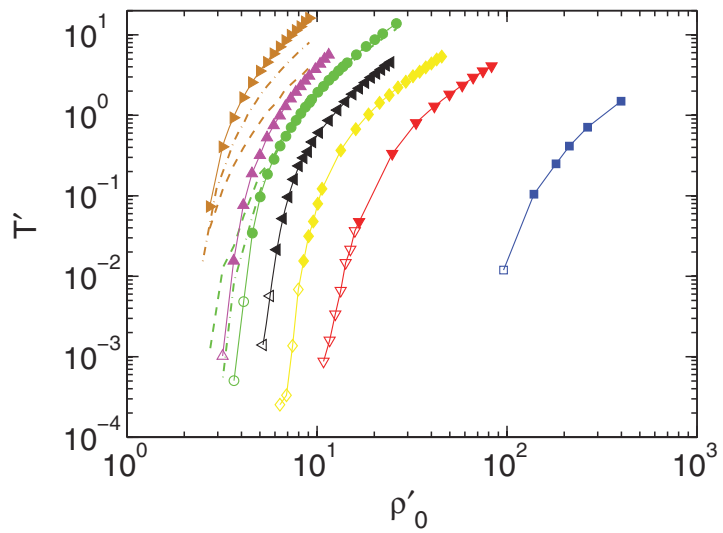

FIG. 8. (Color online) The transmissivity $T^{\prime}$ of the EDZ as a function of $\rho_{0}^{\prime}$. The data are averages over the $x$ and $y$ directions, in 50 random realizations for isotropic networks with various decay lengths $l^{\prime}: l^{\prime}=0.2, L^{\prime}=5$ (solid square), $l^{\prime}=0.5, L^{\prime}=10$ (solid down triangle), $l^{\prime}=1, L^{\prime}=20$ (solid diamond), $l^{\prime}=2, L^{\prime}=20$ (solid left triangle), $l^{\prime}=5, L^{\prime}=20$ (solid circle), $l^{\prime}=10, L^{\prime}=20$ (solid up triangle), $l^{\prime}=1000, L^{\prime}=20$ (solid right triangle). Results for $L^{\prime}=5$ (dashed line) and $L^{\prime}=10$ (dashed-dotted line) are also shown for $l^{\prime}=5$ and 1000. Solid and open symbols correspond to $\rho_{0}^{\prime}$ larger and smaller than $\rho_{0 c}^{\prime}$, respectively. 
In all cases with $l^{\prime} \leqslant 10$, the cell size encompasses a large enough part of the EDZ to consider that the results are its total transmissivity. This is illustrated in Fig. 8 by the comparison of results for $l^{\prime}=5$ with $L^{\prime}=5,10$, and 20 . The finite cell truncates less than $2 \%$ of the total fracture area in the network when $L^{\prime}=20$. In addition, the density in the truncated part is much smaller than in the vicinity of the wall, and therefore its connectivity is poor. Hence, the truncation is even smaller in terms of the contribution to the flow than in terms of fracture area. When $L^{\prime}=10$, about $8 \%$ of the fracture area in the EDZ is truncated, but the results are identical to those for $L^{\prime}=20$ when $\rho_{0}^{\prime} \geqslant 5$, which shows that truncation effects on transmissivity are negligible and suggests that the same applies to the case of $\left(l^{\prime}=10, L^{\prime}=20\right)$ which is truncated in the same proportion. However, a smaller cell with $L^{\prime}=5$ truncates $37 \%$ of the EDZ network and the impact on the transmissivity is visible in Fig. 8. Note also that differences are observed for small values of $\rho_{0}^{\prime}$. They result from the usual finite size effect near the percolation threshold but they do not involve truncation effects since the network beyond $z^{\prime}=5$ is not connected.

Conversely, the network density is nearly uniform when $l^{\prime}=1000$ and the transmissivity obtained in the investigated domain is expected to be proportional to $L^{\prime}$. This is confirmed by the three curves displayed for $L^{\prime}=5,10$, and 20, aside again from a slight size effect near the percolation threshold. This situation is actually not representative of an EDZ, and it is included only as a reference for comparison.

The same data are plotted in Fig. 9 as functions of $\rho_{0}^{\prime}-\rho_{0 c}^{\prime}$. A good representation of the EDZ transmissivity by power laws is possible in all cases, with

$$
T^{\prime}=\alpha_{T}\left(\rho_{0}^{\prime}-\rho_{0 c}^{\prime}\right)^{\beta_{T}} .
$$

The coefficients $\left(\alpha_{T}, \beta_{T}\right)$ given in Table I result from fits of the data in the range of densities where the fraction $\Pi$ of percolating realizations exceeds $98 \%$ (i.e., when size effects due to the proximity of the percolation threshold are negligible).

The exponent $\beta_{T}$ is an increasing function of the density decay length $l^{\prime}$. Note that $\beta_{T}$ is expected to diverge when



FIG. 9. (Color online) The transmissivity $T^{\prime}$ of the EDZ as a function of $\rho_{0}^{\prime}-\rho_{0 c}^{\prime}$, with $\rho_{0 c}^{\prime}$ given by Eq. (33). Same conventions as in Fig. 8. The solid lines are the least-square power-law fits of the data when the fraction $\Pi$ of percolating samples exceeds $98 \%$.



(a)

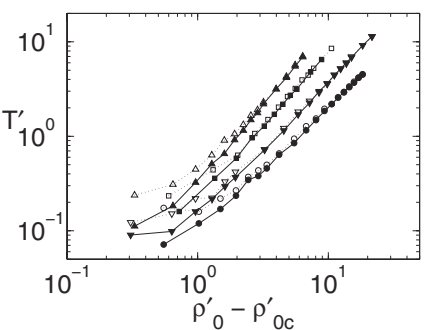

(b)

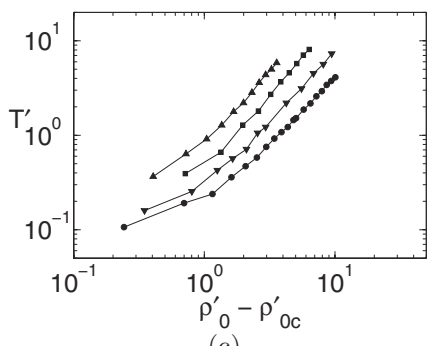

(c)

FIG. 10. The transmissivity $T^{\prime}$ of the EDZ as a function of $\rho_{0}^{\prime}-$ $\rho_{0 c}^{\prime}$, with $\rho_{0 c}^{\prime}$ given by Eq. (33), for networks with $\kappa=0$ (solid circle), 10 (solid down triangle), 50 (solid square), and 200 (solid up triangle). Data are for $l^{\prime}=1, L^{\prime}=5$ (a); $l^{\prime}=2, L^{\prime}=5$ (open symbols), and 10 (solid symbols) (b); and $l^{\prime}=5, L^{\prime}=5$ (c).

$l^{\prime}$ tends to infinity. In this limit, a sudden transition takes place between an impermeable medium when $\rho_{0}^{\prime}<\rho_{c}^{\prime}$ and a permeable medium over an infinite depth (i.e., with infinite $T^{\prime}$ ) when $\rho_{0}^{\prime}>\rho_{c}^{\prime}$. It should be remembered that the data given in Table I for $l^{\prime}=1000$ are for a finite layer $L^{\prime}$ which unlike in all other cases covers only a small part of the EDZ. Hence, $T^{\prime}$ is approximately equal to $L^{\prime} K^{\prime}\left(\rho_{0}^{\prime}\right)$ and accordingly, an exponent 1.82 is obtained which is nearly equal to the exponent 1.80 for $K^{\prime}$ in uniform networks [9].

\section{Anisotropic networks}

The transmissivities obtained in anisotropic networks with concentration parameters $\kappa=10,50$, and 200 are plotted in Fig. 10 as functions of $\rho_{0}^{\prime}-\rho_{0 c}^{\prime}$, where the threshold $\rho_{0 c}^{\prime}$ is obtained by application of Eq. (33).

In Fig. $10(\mathrm{a}), l^{\prime}=1$ and $L^{\prime}=5$ which means that the computation domain contains practically the whole EDZ. Results for $l^{\prime}=2$ are shown in Fig. 10(b) with $L^{\prime}=5$ and 10 . They differ at low density $\left(\rho_{0}^{\prime}-\rho_{0 c}^{\prime} \leqslant 2\right)$ because of size effects near the percolation threshold, but are identical at larger densities which means that the truncation of the EDZ by the finite domain is negligible. Finally, results for $l^{\prime}=5$ with $L^{\prime}=5$ are plotted in Fig. 10(c). These data are certainly affected by truncation effects.

In all cases, the transmissivity increases with the degree of anisotropy. The evolution of $T^{\prime}$ with the density remains governed by power laws of the type of (34), with the exponent $\beta_{T}$ a slightly increasing function of $\kappa$.

\section{MODEL FOR THE EDZ TRANSMISSIVITY}

\section{A. Parallel flow model}

The principle of the model runs as follows. The flow properties of the EDZ vary with the distance $z$ from the wall. 
However, the macroscopic pressure gradient does not depend on $z$, and the flow lines are in average parallel to the wall. Hence, the overall transmissivity is tentatively estimated by a parallel flow model, where a layer at depth $z$ behaves as a fractured medium with uniform properties corresponding to the state at this position in the EDZ. Accordingly,

$$
\widehat{T}^{\prime}=\int_{0}^{L_{c}^{\prime}} K^{\prime}\left(z^{\prime}\right) d z^{\prime}
$$

This implicitly assumes that the medium properties are slowly varying (i.e., that $l^{\prime}$ is large). Hence, this model can be regarded as the leading order contribution in a power series expansion in terms of $1 / l^{\prime}$. The integral in Eq. (35) is truncated beyond the distance $L_{c}^{\prime}$ from the wall where the density becomes smaller than the percolation threshold $\rho_{0 c}^{\prime}$. The local permeability $K^{\prime}\left(z^{\prime}\right)$ is evaluated by application of Eqs. (29) and (30). This yields

$$
\widehat{T}^{\prime}=\frac{\psi_{\perp}}{\Phi} \int_{0}^{L_{c}^{\prime}} \frac{\alpha\left(\rho^{\prime}-\rho_{c}^{\prime}\right)^{2}}{1+\beta\left(\rho^{\prime}-\rho_{c}^{\prime}\right)} d z^{\prime}=l^{\prime} \frac{\psi_{\perp}}{\Phi} \mathcal{J}
$$

with

$$
\mathcal{J}=\int_{\rho_{0 c}^{\prime}-\rho_{c}^{\prime}}^{\rho_{0}^{\prime}-\rho_{c}^{\prime}} \frac{\alpha u^{2}}{(1+\beta u)\left(\rho_{c}^{\prime}+u\right)} d u
$$

The second form results from the change of variable $u=\rho^{\prime}-$ $\rho_{c}^{\prime}$. An expression for the correction factor $\Phi$ as a function of $\kappa$ and $l^{\prime}$ has been provided in (9). Recall that $l^{\prime}$ should be replaced by $l_{A}^{\prime}(10)$ if the fractures are not circular. The expression (28) for $\psi_{\perp}$ in uniform networks, which results from the calculation of the average $\langle\mathbf{I}-\mathbf{n n}\rangle$ in Eq. (27) can be generalized for finite $l^{\prime}$. It was obtained in [10] as

$$
\psi_{\perp}=\frac{3}{2} l^{\prime 2} \frac{\kappa}{\sinh \kappa}\left[\left(1+\frac{\kappa^{2}}{E^{2}}\right) \cosh E-2 \cosh \kappa+\frac{\sinh E}{\ell^{\prime 2} E^{3}}\right] .
$$

This expression for circular fractures was shown to apply to other fracture shapes as well if $l^{\prime}$ is replaced by $l_{A}^{\prime}$.

The predictions of the model are compared to the numerical data for isotropic networks in Fig. 11(a). Note that in some of the cases listed in Table I with small values of $L^{\prime}$ the EDZ is truncated. For $l^{\prime}=1000$, the sample actually contains only a slab of nearly uniform medium. These truncation effects are easily accounted for in the model by setting the upper bound of the integral in Eq. (35) to $L^{\prime}$ instead of $L_{c}^{\prime}$. This has been done for $l^{\prime}=1000$ and in the case of $l^{\prime}=L^{\prime}=5$ which is also shown for illustration in Fig. 11(a).

The agreement of the comparison is very satisfactory, considering the simplicity of the argument. Significant deviations are observed only for steep density decays with $l^{\prime}=0.5$ and especially 0.2. This is not surprising since endowing the network with local mean properties when its characteristics vary on a scale much smaller than the fracture size is obviously risky. The general requirement for any homogenization process of a separation between the micro- and macroscopic scales is not fulfilled. Fortunately, $l^{\prime}$ is generally not smaller than one in real situations.

There might be another reason for these deviations in this range of $l^{\prime}$. Equation (36) results from Eq. (35) with $K^{\prime}\left(z^{\prime}\right)$ set equal to the permeability of a uniform network with the same

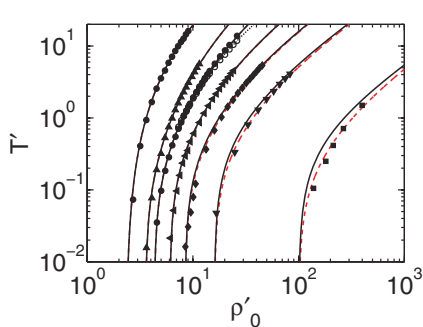

(a)

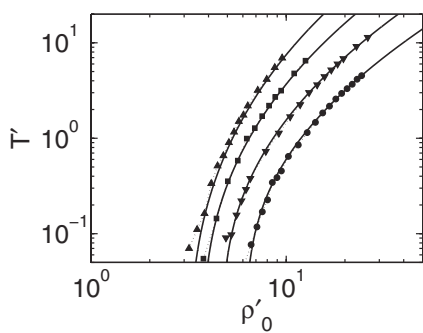

(c)

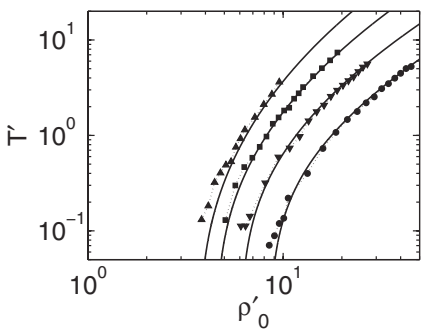

(b)

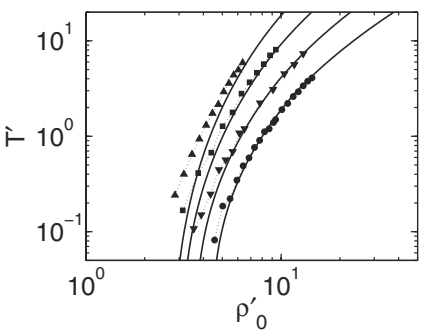

(d)
FIG. 11. (Color online) The transmissivity $T^{\prime}$ of the EDZ as a function of $\rho_{0}^{\prime}$. (a) Isotropic networks with various $l^{\prime}$. The symbols are the numerical data, with the same conventions as in Fig. 8. (b)-(d) Anisotropic networks with various $\kappa$. The symbols are the numerical data, with the same conventions as in Fig. 10. Data are for $l^{\prime}=$ $1, L^{\prime}=5(\mathrm{~b}) ; l^{\prime}=2, L^{\prime}=10(\mathrm{c})$; and $l^{\prime}=5, L^{\prime}=5$ (d). In all cases, the black solid lines are the model (36). The red dashed lines in (a) are the model (38) for $l^{\prime} \leqslant 1$. The open symbols and the dotted line in (a) correspond to $l^{\prime}=5, L^{\prime}=5$.

$\rho^{\prime}$, which is a measure of the degree of fracturation in terms of the network connectivity. A natural alternative is to use the permeability of the uniform network with the same local volumetric area of fracture $\mathcal{S}$, which has a density $\psi_{S} \rho^{\prime} / \Phi$. The two procedures are equivalent when $l^{\prime} \geqslant 1$, since $\psi_{S} / \Phi$ is then nearly unity, but they are not for $l^{\prime}<1$. An alternative model can be proposed in these terms as

$$
\widehat{T}_{S}^{\prime}=l^{\prime} \frac{\psi_{\perp}}{\psi_{S}} \int_{\frac{\psi_{S}}{\Phi} \rho_{0 c}^{\prime}-\rho_{c}^{\prime}}^{\frac{\psi_{S}}{\Phi} \rho_{0}^{\prime}-\rho_{c}^{\prime}} \frac{\alpha u^{2}}{(1+\beta u)\left(\rho_{c}^{\prime}+u\right)} d u .
$$

$\widehat{T}^{\prime}{ }_{S}$ is also plotted in Fig. 11(a) and seen to be in much better agreement with the numerical data for small $l^{\prime}$.

The predictions of the model (36) for anisotropic networks are compared in Figs. 11(b)-11(d) to the numerical data. Again, a very good agreement is observed. For $l^{\prime}=1$ [Fig. 11(b)], deviations are observed only for low densities and they are due to finite size effects. Although $L^{\prime}=5$ is sufficient to avoid truncation of the EDZ, it is too small to eliminate the size effects near the percolation threshold. These deviations are much smaller in Fig. 11(c) for $l^{\prime}=2$, since the cell size $L^{\prime}=10$ is larger. In the last case with $l^{\prime}=5$ [Fig. 11(d)], the deviations are more significant, especially for the very anisotropic networks with $\kappa=200$. However, these results should be considered with caution, since the small cell size $L^{\prime}=5$ induces both percolation size effects and a severe truncation of the EDZ.

Note that model (38) is unsuccessful for anisotropic networks. Whereas Eqs. (36) and (38) are equivalent for isotropic networks when $l^{\prime} \geqslant 1$ because $\psi_{S} / \Phi \approx 1$, this ratio becomes very large when $\kappa$ increases and Eq. (38) strongly 
deviates from the numerical data. Hence, the interest of the alternative model based on the volumetric fracture area in its current form (38) is restricted to isotropic networks with $l^{\prime}<1$. For this reason, only model (36) is considered in the rest of the discussion.

\section{B. Discussion}

Although the parallel flow model (36) is only valid for large $l^{\prime}$, its success in representing the numerical data makes it a convenient framework for the description of general trends.

Notice first that in the two limits where $\rho_{0}^{\prime}$ is very close to or much larger than $\rho_{0 c}^{\prime}$ the integral $\mathcal{J}$ in Eq. (36b) becomes a linear function of $\rho_{0}^{\prime}-\rho_{0 c}^{\prime}$. This is because the difference $\rho_{0}^{\prime}-\rho_{0 c}^{\prime}$ between the upper and lower bounds for the integral is vanishingly small in the first case, and because the integrand tends to the constant value $\alpha / \beta$ for $\rho_{0}^{\prime} \gg \rho_{0 c}^{\prime}$ in the second case. The asymptotic regimes are

$$
\begin{aligned}
& \widehat{T}^{\prime} \approx l^{\prime} \frac{\psi_{\perp}}{\Phi} \frac{\widehat{K}^{\prime}\left(\rho_{0 c}^{\prime}\right)}{\rho_{0 c}^{\prime}}\left(\rho_{0}^{\prime}-\rho_{0 c}^{\prime}\right), \quad\left(\rho_{0}^{\prime}-\rho_{0 c}^{\prime} \ll 1\right), \\
& \widehat{T}^{\prime} \approx l^{\prime} \frac{\psi_{\perp}}{\Phi} \frac{\alpha}{\beta}\left(\rho_{0}^{\prime}-\rho_{0 c}^{\prime}\right), \quad\left(\rho_{0}^{\prime} \gg \rho_{0 c}^{\prime}\right) .
\end{aligned}
$$

A transition takes place between these two regimes for intermediate $\rho_{0}^{\prime}$, as can be seen in Fig. 12. The slopes of the curves (in log-log plots) at the inflexion points range from 1.0 for very small $l^{\prime}$ to 2.0 for $l^{\prime} \sim 10^{2}$ and 2.5 for $l^{\prime} \sim 10^{3}$. These apparent power-law behaviors with increasing exponents correspond with the observations in Fig. 9, whose range covers the central part of Fig. 12. Note that in the limit of infinite $l^{\prime}$, the EDZ becomes a semi-infinite half space with uniform properties and its transmissivity is zero or infinite when the density is smaller or larger than $\rho_{0 c}^{\prime}=\rho_{c}^{\prime}$, respectively. This explains the steepening of the transitions in the curves in Fig. 12 for large $l^{\prime}$. The value 2.5 observed for $l^{\prime}=10^{3}$ is larger than the value $\beta_{T}=1.82$ in Table I because $\widehat{T}^{\prime}$ gives the transmissivity of the whole EDZ whereas the

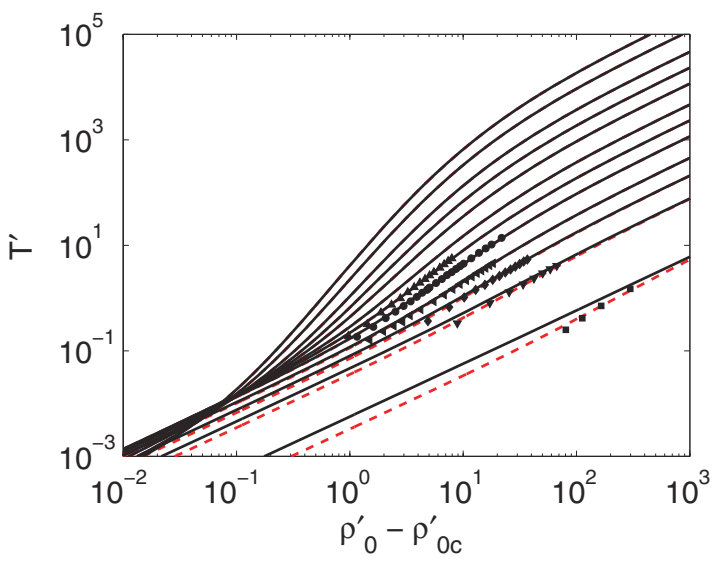

FIG. 12. (Color online) The models (36b) and (38) for the EDZ transmissivity of isotropic networks, as a function of $\rho_{0}^{\prime}-\rho_{0 c}^{\prime}$, for $l^{\prime}=0.2,0.5,1,2,5,10,20,50,100,200,500$, and 1000 (bottom to top). Black solid lines correspond to Eq. (36b); red dashed lines correspond to Eq. (38). The symbols are the numerical data from Fig. 9 when the fraction $\Pi$ of percolating samples exceeds $98 \%$.



(a)

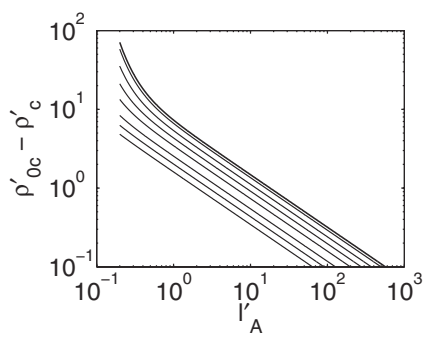

(c)

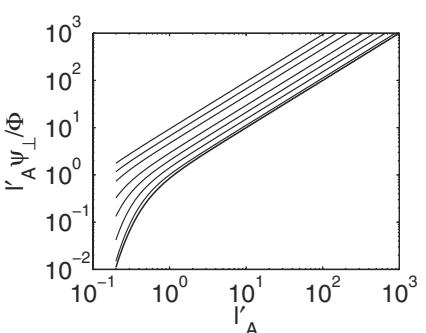

(b)

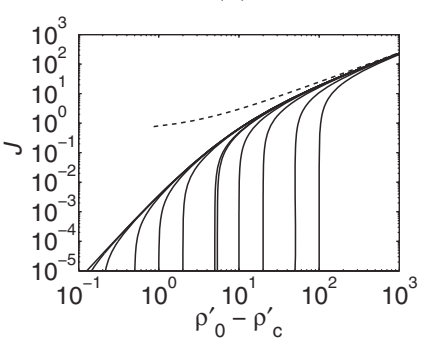

(d)
FIG. 13. (a) The transmissivity $\widehat{T}^{\prime}$ [Eq. (36b)] for isotropic networks as a function of $\rho_{0}^{\prime}-\rho_{c}^{\prime}$, for $l_{A}^{\prime}=0.2,0.5,1,2,5,10$, 20,50,100, 200, 500, and 1000 (bottom to top). (b) The prefactor $l^{\prime} \psi_{\perp} / \Phi$ in (36b) as a function of $l_{A}^{\prime}$ for $\kappa=0,2,5,10,20,50,100$, 200 (bottom to top). (c) The lower bound $\rho_{0 c}^{\prime}-\rho_{c}^{\prime}$ of the integral $\mathcal{J}$ in Eq. (36b) as a function of $l_{A}^{\prime}$ for $\kappa=0,2,5,10,20,50,100,200$ (top to bottom). (d) The integral $\mathcal{J}$ in Eq. (36b) as a function of $\rho_{0}^{\prime}-\rho_{c}^{\prime}$ for $\rho_{0 c}^{\prime}-\rho_{c}^{\prime}=0,0.1,0.2,0.5,1,2,5,10,20,50,100$ (left to right). The broken line in (d) is the upper bound (40).

data for $l^{\prime}=10^{3}$ in Fig. 9 are for a finite slab with $L^{\prime}=20$. Therefore, their variations result only from the increase of the integrand in Eq. (35) and do not account for the increase of the upper bound $L_{c}^{\prime}$ of the integral.

The large density limit [Eq. (39b)] is an interesting result. It is an upper bound for $\widehat{T}^{\prime}$, since it is obtained by replacing the integrand in Eq. (36b) by its large density limit $\alpha / \beta$, which is a majorant. If one neglects $\rho_{0 c}^{\prime}$ with respect to $\rho_{0}^{\prime}$, Eq. (39b) reduces to

$$
\widehat{T^{\prime}} \approx l^{\prime} \frac{k_{S} \rho_{0}^{\prime} \psi_{\perp}}{\Phi}, \quad\left(\rho_{0}^{\prime} \gg 1\right) .
$$

Hence, an upper bound for the transmissivity of the EDZ, which is also a reasonable first estimate at very large densities, is obtained by multiplying Snow's permeability value [Eq. $(27 \mathrm{c})]$ based on the density $\rho_{0}^{\prime}$ near the wall by the decay length $l^{\prime}$.

If model (38) is used instead of Eq. (36b), Eq. (39b) is unchanged but Eq. (39a) is multiplied by $\Phi / \psi_{S}$. The model predictions are shown in Fig. 12. As already seen in Fig. 11(a), they are in better agreement with the numerical data than Eq. (36b) when $L^{\prime}<1$.

\section{Graphical tools}

Although all the analytical formulae have been given for the evaluation of the transmissivity $\widehat{T}^{\prime}$ by the model (36b), it might be useful to provide as well graphical tools from which a first estimate can be quickly and easily obtained. 
This is easy for isotropic networks, since $\widehat{T}^{\prime}$ is a function only of $l_{A}^{\prime}$ and $\rho_{0}^{\prime}$. The very small variations of $\rho_{c}^{\prime}$ around 2.3 according to the fracture shape are neglected here. $\widehat{T}^{\prime}$ as given by Eq. (36b) is plotted in Fig. 13(a) as a function of $\rho_{0}^{\prime}-\rho_{c}^{\prime}$, for a series of values of $l_{A}^{\prime}$.

For anisotropic networks, $\widehat{T}^{\prime}$ depends on $l_{A}^{\prime}, \kappa$, and $\rho_{0}^{\prime}$ and several steps are required. First, the prefactor $l^{\prime} \psi_{\perp} / \Phi$ is plotted in Fig. 13(b) as a function of $l_{A}^{\prime}$ for a series of values of $\kappa$. Then, the lower bound $\rho_{0 c}^{\prime}-\rho_{c}^{\prime}$ of the integral $\mathcal{J}$ in Eq. (36b) is given in Fig. 13(c). Finally, the integral $\mathcal{J}$ is plotted in Fig. 13(d) as a function of $\rho_{0}^{\prime}-\rho_{c}^{\prime}$, for a series of values of the lower bound $\rho_{0 c}^{\prime}-\rho_{c}^{\prime}$ [obtained from Fig. 13(c)].

Let us give an example. Suppose $l_{A}^{\prime}=1$ and $\kappa=5$. Figure 13(b) yields directly the prefactor $l^{\prime} \psi_{\perp} / \Phi \approx 1.4$ and Fig. 13(b) gives $\rho_{0 c}^{\prime}-\rho_{c}^{\prime} \approx 5$.4. Then, if $\rho_{0}^{\prime}=10$, the value of $\mathcal{J}$ is read in Fig. 13(d) on the curve $\rho_{0 c}^{\prime}-\rho_{c}^{\prime}=5$ (or slightly below) at the position $\rho_{0}^{\prime}-\rho_{c}^{\prime}=10-2.3=7.7$, which yields $\mathcal{J} \approx 0.23$. Thus, $\widehat{T}^{\prime} \approx 1.4 \times 0.23 \approx 0.32$, or dimensionally $T \approx 0.32 \sigma$. The exact result is $\widehat{T}^{\prime}=0.29$.

\section{CONCLUDING REMARKS}

A systematic investigation of the percolation and flow properties of the fracture network in an EDZ along a gallery wall has been conducted. The main parameters are the fracturation density $\rho_{0}^{\prime}$ near the wall, its exponential decay length $l^{\prime}$ within the embedding rock, and possibly the concentration parameter $\kappa$ in the case of an anisotropic Fisher distribution orientation of the fractures.

Heuristic formulas (33) and (36) have been proposed which successfully account for the numerical results, both for the percolation threshold and for the hydraulic transmissivity.

Additional work is underway for a better substantiation of the parallel flow model (36). It includes a detailed examination of the flow density in the EDZ as a function of the distance from the wall.

Aside from the Fisher distribution, other anisotropic orientation distributions can be observed, such as two conjugated families of fractures at opposite angles with the normal to the gallery wall. It would be an interesting extension to repeat some of the steps taken here in this situation. This involves the calculation of some geometrical functions $\left(\Phi, \psi_{c}, \psi_{S}, \psi_{\perp}\right)$, along the same lines as in Ref. [10], and the determination of the percolation threshold for such an EDZ. It can be expected that a power law of the kind of Eq. (33) is still applicable, as well as the parallel flow model (36) for the transmissivity. This would considerably extend the scope of this model, and reduce the prediction of the EDZ transmissivity to the evaluation of a few geometrical functions which account for the spatial density variation rate and for the orientational characteristics of the network.
[1] O. Huseby, J.-F. Thovert, and P. M. Adler, J. Phys. A 30, 1415 (1997).

[2] N. Koudina, R. Gonzalez Garcia, J.-F. Thovert, and P. M. Adler, Phys. Rev. E 57, 4466 (1998).

[3] V. V. Mourzenko, J.-F. Thovert, and P. M. Adler, Phys. Rev. E 69, 066307 (2004).

[4] V. V. Mourzenko, J.-F. Thovert, and P. M. Adler, Phys. Rev. E 72, 036103 (2005).

[5] I. I. Bogdanov, V. V. Mourzenko, J.-F. Thovert, and P. M. Adler, Water Resourc. Res. 39, 1023 (2003).

[6] I. I. Bogdanov, V. V. Mourzenko, J.-F. Thovert, and P. M. Adler, Phys. Rev. E 76, 036309 (2007).

[7] M. Khamforoush, K. Shams, J.-F. Thovert, and P. M. Adler, Phys. Rev. E 77, 056307 (2008).

[8] V. Mourzenko, J.-F. Thovert, P. M. Adler, in Proceedings of the International Conference on Rock Joints and Jointed Rock Masses, Tucson, 2009, edited by Pinnaduwa H.S.W. Kulatilake (University of Arizona, Tucson, 2009).

[9] V. V. Mourzenko, J.-F. Thovert and P. M. Adler, Phys. Rev. E 84, 036307 (2011).
[10] V. V. Mourzenko, J.-F. Thovert, and P. M. Adler, Phys. Rev. E 83, 031104 (2011).

[11] P. Bossart, P. M. Meier, A. Moeri, T. Trick, and J.-C. Mayor, Eng. Geol. 66, 19 (2002).

[12] J.-F. Thovert, V. V. Mourzenko, P. M. Adler, C. Nussbaum, and P. Pinettes, Eng. Geol. 117, 39 (2011).

[13] K. V. Mardia, Statistics of Directional Data (Academic Press, London, 1972).

[14] I. Balberg, C. H. Anderson, S. Alexander, and N. Wagner, Phys. Rev. B 30, 3933 (1984).

[15] P. M. Adler and J.-F. Thovert, Fractures and Fracture Networks (Kluwer Academic Publishers, Dordrecht, 1999).

[16] J.-F. Thovert and P. M. Adler, Geoph. Res. Lett. 31, L22502 (2004).

[17] D. Stauffer and A. Aharony, Introduction to Percolation Theory (Taylor and Francis, Bristol, 1992).

[18] D. T. Snow, Water Resour. Res. 5, 1273 (1969).

[19] H. Alkan, Int. J. Rock Mech. \& Mining Sciences 46, 716 (2009).

[20] P. Sotta and D. Long, Eur. Phys. J. E 11, 375 (2003). 